\title{
Asymmetrical Cross-Sectional Buckling in Arc-Prepared Multiwall Carbon Nanotubes Revealed by Iodine Filling
}

\author{
Abraao Cefas Torres-Dias ${ }^{1}$, Anthony Impellizzeri ${ }^{2}{ }^{(D}$, Emmanuel Picheau $^{3}\left(\mathbb{D}\right.$, Laure Noé $^{1}$, Alain Pénicaud ${ }^{3}$ (D), \\ Christopher Ewels ${ }^{2, *}$ and Marc Monthioux $1, *$ (D)
}

Citation: Torres-Dias, A.C.; Impellizzeri, A.; Picheau, E.; Noé, L.; Pénicaud, A.; Ewels, C.; Monthioux, M. Asymmetrical Cross-Sectional Buckling in Arc-Prepared Multiwall Carbon Nanotubes Revealed by Iodine Filling. C 2022, 8, 10. https://doi.org/10.3390/c8010010

Academic Editor: Craig E. Banks

Received: 20 December 2021

Accepted: 19 January 2022

Published: 27 January 2022

Publisher's Note: MDPI stays neutral with regard to jurisdictional claims in published maps and institutional affiliations.

Copyright: (C) 2022 by the authors. Licensee MDPI, Basel, Switzerland. This article is an open access article distributed under the terms and conditions of the Creative Commons Attribution (CC BY) license (https:// creativecommons.org/licenses/by/ $4.0 /)$.
1 Centre d'Elaboration des Matériaux et d'Etudes Structurales (CEMES), UPR8011 CNRS, Université de Toulouse, CEDEX 04, 31055 Toulouse, France; abraaocefas@gmail.com (A.C.T.-D.); noe@cemes.fr (L.N.)

2 Institut des Matériaux de Nantes Jean Rouxel (IMN), UMR-6502 CNRS, Université de Nantes, 44000 Nantes, France; anthony.Impellizzeri@cnrs-imn.fr

3 Centre de Recherche Paul Pascal (CRPP), UMR5031 CNRS, Université de Bordeaux, 33600 Pessac, France; emmanuel.picheau@crpp.cnrs.fr (E.P.); alain.penicaud@crpp.cnrs.fr (A.P.)

* Correspondence: chris.ewels@cnrs-imn.fr (C.E.); marc.monthioux@cemes.fr (M.M.)

\begin{abstract}
We report the intercalation of iodine chains in highly crystalline arc-discharge multiwalled carbon nanotubes (MWCNTs), not in the central cavity but instead between the concentric graphene shells. High-resolution transmission electron microscopy demonstrated that the intercalation was asymmetric with respect to the longitudinal axis of the nanotubes. This filling is explained through the existence of asymmetric intershell channels which formed as the tubes shrank upon cooling after growth. Shrinkage occurred because the geometrically constrained equilibrium intershell spacing was higher at growth than room temperature, due to the highly anisotropic coefficient of thermal expansion of graphite (or graphene stacks). Computational modelling supported the formation of such cavities and explained why they all formed on the same side of the tubes. The graphene shells were forced to bend outward, thereby opening aligned intergraphene nanocavities, and subsequently allowing the intercalation with iodine once the tube ends were opened by oxidative treatment. These observations are specific to catalyst-free processes because catalytic processes use too low temperatures, but they are generally applicable in geometrically closed carbon structures grown at high temperatures and so should be present in all arc-grown MWCNTs. They are likely to explain multiple observations in the literature of asymmetric interlayer spacings in multiple-shell graphenic carbon structures.
\end{abstract}

Keywords: multiwalled carbon nanotube; electric arc; texture; iodine intercalation; transmission electron microscopy; modelling; coefficient of thermal expansion

\section{Introduction}

Concentric MWCNTs are tubular structures composed of multiple rolled-up graphene shells, having nanosized outer and inner diameters in the range of a few nanometres to tens of nanometres [1]. Although electric arc plasma was shown to be an efficient way to form MWCNTs [2], most MWCNTs are now prepared by low-temperature, catalyst-assisted processes (the thermal cracking of hydrocarbon molecules, or $\mathrm{CO}$ disproportionation), which historically were actually the first ways to produce MWCNTs [3]. The major drawback of the electric arc process is that it is poorly adapted to bulk and/or continuous production and allows little control over the MWCNT length, number of concentric shells, and growth orientation. On the other hand, it produces MWCNT powder free of any catalyst and amorphous carbon content, and most of all, the quality of the graphene nanotexture within the CNT wall is much higher than in catalyst-based processes (except for MWCNTs with very few shells). The reason for this is that arc-prepared MWCNTs grow at very high temperatures (several thousands of degrees) from elemental carbon species (single atoms or dimers) originating from a sublimed graphite anode (the smaller the bricks, the higher the 
chances they arrange correctly), with no heteroatom around to interfere (generally, apart from $\mathrm{He}$, needed to generate the plasma) [4]. Basically, in the recombination process of the carbon species, the graphene network is preferred because of the thermodynamics, the elongated morphology as a filament is preferred because of the electric field, and the cylindrical morphology of the graphenes is preferred in order to minimise the dangling bonds.

CNT properties can be modified by filling with properly selected materials $[5,6]$, and the effect on the properties is all the more pronounced when the number of graphenes in the tube wall is small. For instance, as fillers, water or iodine molecules increase the mechanical stability [7]. However, the most remarkable effects are related to electronic doping. For example, Cs [8] and K [9] act as electron donors, while bromine and iodine work as electron acceptors [10]. As an application of the latter, fibres composed of DWCNTs filled (therefore doped) with iodine species have their electrical conductivity increased from $1.1 \mathrm{MS} . \mathrm{m}^{-1}$ (undoped DWCNTs) to $6.5 \mathrm{MS} . \mathrm{m}^{-1}$, increasing the specific electrical conductivity above that of copper [11]. Additionally, filling CNTs with ferroelectric materials such as Co [12], Fe [13], and Ni is expected to be a means to control the electronic transport by applying external electromagnetic fields. Examples of applications that CNTs doped in various ways may be suitable for can be found in [14].

As a continuation of the work aiming at closely combining nanotubes and heteroelements or phases by filling the former with the latter, we intended to fill the lateral channels of flattened CNTs [15] with iodine. However, nature does what it wants regardless of what the experimentalists intend, and our first attempts failed. Nevertheless, interesting results were obtained in the meanwhile, regarding the behaviour of the filling material (iodine) with respect to regular MWCNTs prepared by the electric arc-discharge method and then subjected to an oxidation process. We report in this paper the transmission electron microscopy observations of the resulting materials, which revealed a textural feature specific to arc-prepared MWCNTs, unrevealed to date. Our interpretations are compared with the literature and supported by calculations.

\section{Experimental and Computational Methods}

\subsection{Materials}

Arc-discharge MWCNTs were acquired from both MER Corporation and Sigma Aldrich. Figure 1 provides some examples of both nanotube batches. Their description is consistent with that of the literature [2,16-19], i.e., they are made with perfect concentric graphene shells whose defects are mostly concentrated at both MWCNT ends or distributed along the MWCNTs to form bamboo-like transverse walls. Defects responsible for this are heterocycles (rings with 5 or 7 carbon atoms instead of 6) which induce positive or negative local curvatures, finally allowing the formation of terminal caps. Some polygonisation of the transverse cross-section of the MWCNTs making the tube body facetted was suggested [17], mostly because of the comparison made with the obviously polyhedral multiwall carbon shells which are formed along with the tubes. However, direct demonstration providing clear evidence of this is lacking, and our discussion will actually state that this case is unlikely.

The as-received MWCNTs were thermally oxidised down to $70 \%$ weight loss, either in a thermogravimetric analysis (TGA) machine (TA Q50 TA instrument, $10 \mathrm{mg}$ at $590{ }^{\circ} \mathrm{C}$, dry air conditions) or in a home-made, open-air cylindrical oven $\left(300 \mathrm{mg}\right.$ at $\left.580^{\circ} \mathrm{C}\right)$. Both procedures allow the burning away of most of the carbon particles and opening of many of the nanotube ends (Figure 2).

Then, $30 \mathrm{mg}$ of the powder remaining from the oxidation step in the cylindrical oven was sonicated for $1 \mathrm{~h}$ in $100 \mathrm{~mL}$ of water containing $1 \mathrm{~g}$ of sodium cholate (Sigma Aldrich, Schnelldorf, Germany) using a Digital Sonifier 450 (Branson, Rungis, France) equipped with a $1 / 2$ " diameter titanium tip at $62 \%$ of the maximum amplitude (crenel $0.5 \mathrm{~s}$ ON and $0.2 \mathrm{~s}$ OFF). The surfactant was then washed away using $2-3$ successive ultracentrifugation steps ( $5 \mathrm{~min}$ at 50,000 $\mathrm{g}$, then $5 \mathrm{~min}$ at 100,000 g, and then $20 \mathrm{~min}$ at 150,000 g) and replacement of the supernatant by deionised water between each centrifugation step in order to manually 
redisperse the powder at the bottom of the centrifugation containers. The remaining traces of surfactant were washed away by dialysis until the electrical conductivity of the solution reached that of deionised water (dialysis membrane of 5-7 kDA). The solution was then lyophilised to obtain a powder.

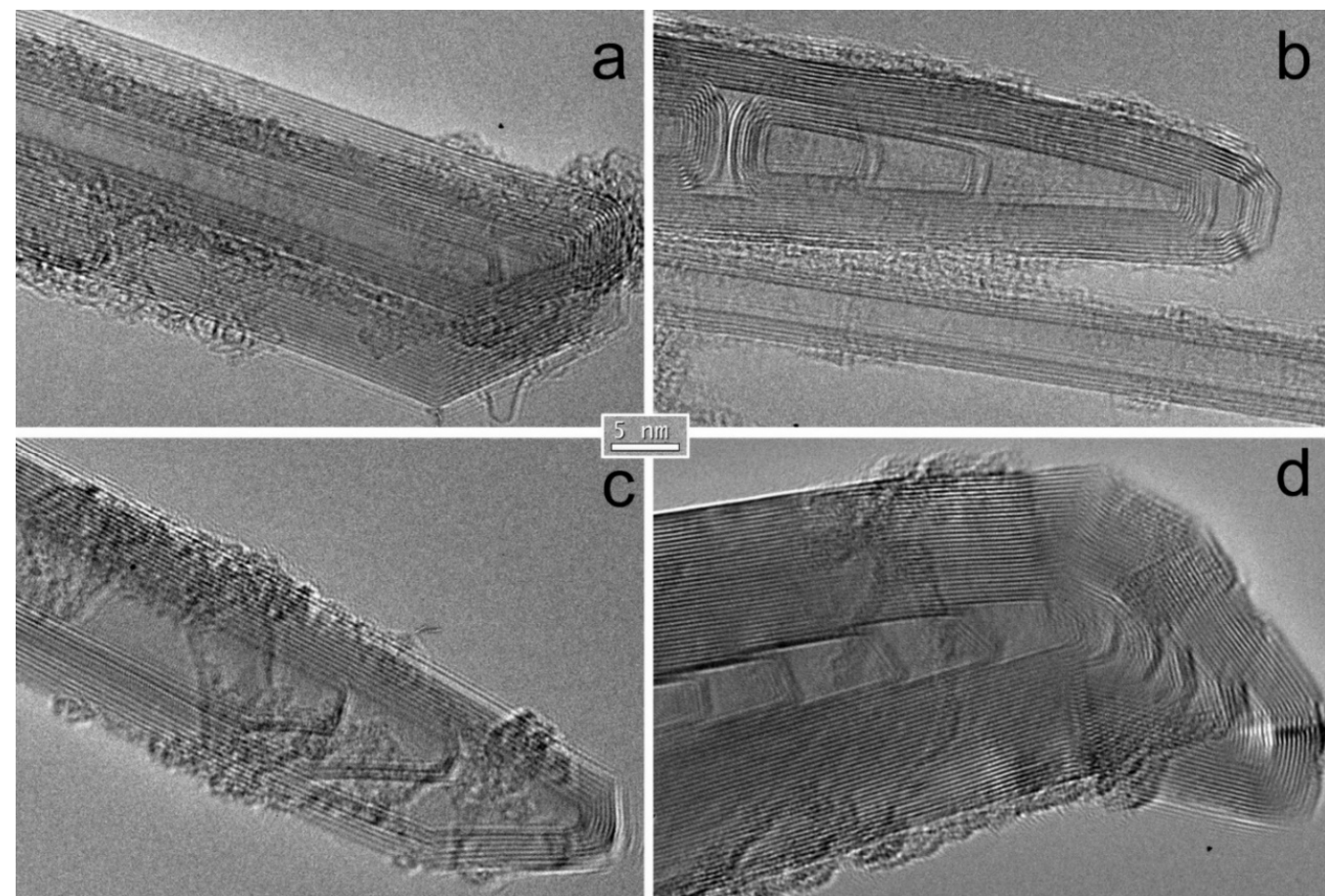

Figure 1. HRTEM images of ends of as-received, arc-prepared MWCNTs from two different commercial batches: (a,b) from MER Corporation; (c,d) from Sigma Aldrich.
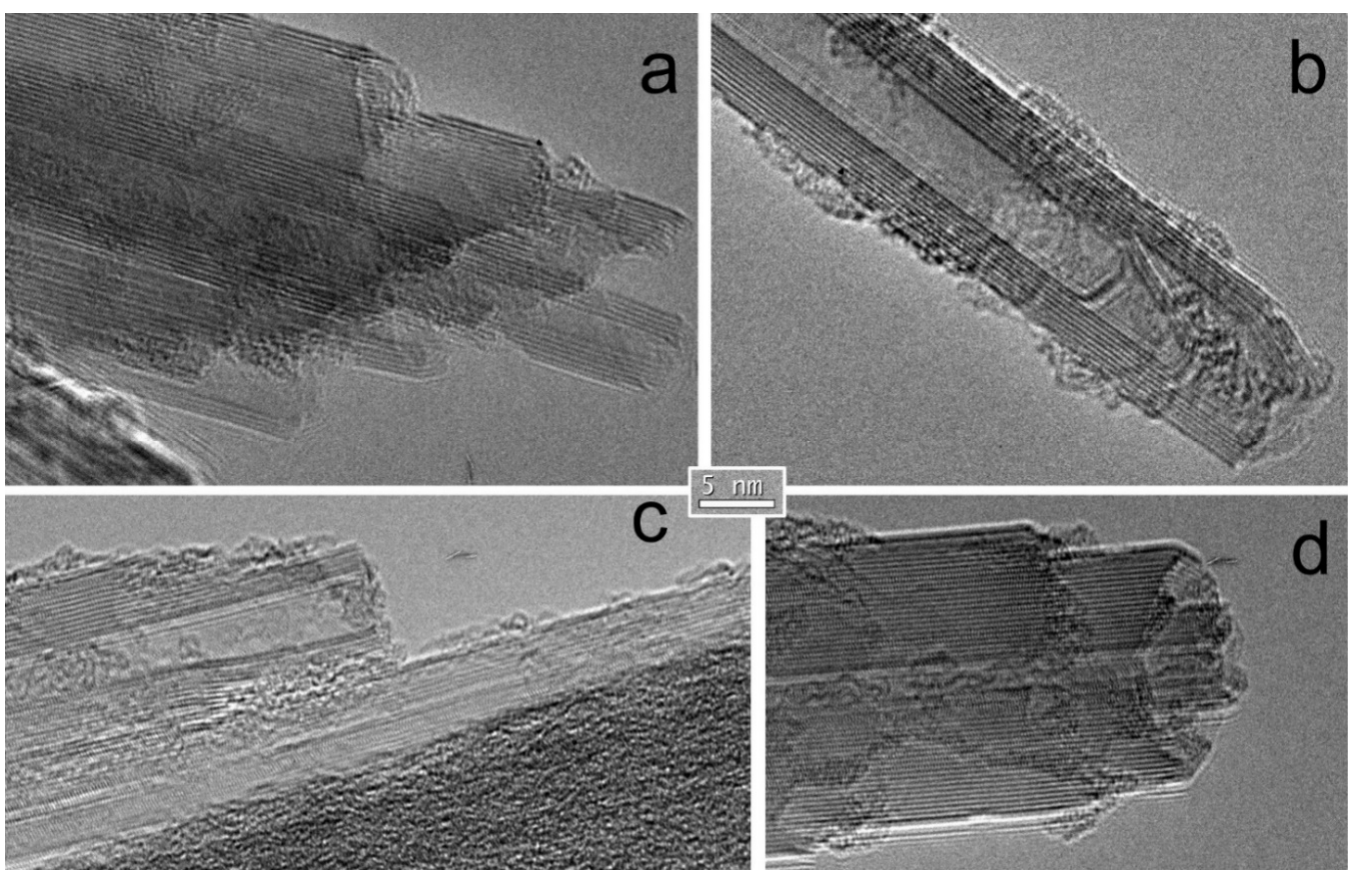

Figure 2. HRTEM images of ends of oxidised (in TGA), arc-prepared MWCNTs from the same commercial batches as in Figure 1: $(\mathbf{a}, \mathbf{b})$ from MER Corporation; $(\mathbf{c}, \mathbf{d})$ from Sigma Aldrich.

The side-nanochannels of the ribbon-like flattened tubes mentioned in the Introduction (Section 1) were initially intended to be filled with iodine. The filling procedure followed 
that reported in [20]. Only the MER Corporation MWCNTs were subjected to this procedure. Powdered MWCNTs $(10 \mathrm{mg})$ and solid chips of iodine $(275 \mathrm{mg})$ were mixed (1:1.3 molar mass ratio), then ground in a mortar until visual homogeneity could be observed. The mixture was sealed in a glass tube, then filled with argon and vacuumed three times. The glass tube was sealed under vacuum and then heated at $5{ }^{\circ} \mathrm{C} / \mathrm{min}$ up to $144{ }^{\circ} \mathrm{C}$. The temperature was kept constant for $24 \mathrm{~h}$. Then, the glass tube was cooled down to room temperature at $1{ }^{\circ} \mathrm{C} / \mathrm{min}$. The content was recovered from the glass tube and washed with absolute ethanol over a $20 \mathrm{~nm}$ pore filter to remove excess iodine. The resulting content was stored in absolute ethanol $(20 \mathrm{~mL})$.

\subsection{Transmission Electron Microscopy}

Before transmission electron microscopy (TEM) investigation, all the materials of interest were gently dispersed in absolute ethanol by bath sonication (room temperature, 120 s). Each dispersion was then drop-casted over copper TEM grids coated with a lacey amorphous carbon film. High-resolution (HR) bright-field TEM was performed to reveal the lattice fringes using either a FEI TECNAI F20 with the objective lens corrected from the spherical aberration and operated at $100 \mathrm{kV}$, or a JEOL ARM with the condenser lenses corrected from the spherical aberration and operated at $200 \mathrm{kV}$, while taking care not to overexpose the material to the electron flow in order to minimise irradiation damaging. The JEOL ARM was also used to perform High-Angle Annular Dark-Field (HAADF) STEM imaging, as it provides an enhanced contrast based on atomic numbers, with the heavier the brighter. It therefore allowed us to clearly distinguish between $C(Z=6)$ and I $(Z=53)$ elements [21].

\subsection{Computational}

Calculations are carried out within density functional theory (DFT) as implemented in AIMPRO software package [22-24]. The generalised gradient approximation (GGA) with Perdew, Burke, and Ernherzolf parametrisation was used throughout. To take into account van der Waals (vdW) interactions, the Grimme D2 scheme was adopted to treat long-range interatomic dispersion interaction [25]. The electron wave functions were expanded using a basis of relativistic Gaussian function sets multiplied by polynomial functions including angular momenta up to maxima $p(l=0,1), d(l=0-2)$, and $f(l=0-3)$. Carbon and iodine were treated using $p d p p$ and $d d d d$ basis sets, giving 22 and 40 independent functions per atom, respectively. A system-dependent plane-wave energy cut-off of $175 \mathrm{Ha}$ (where $\mathrm{Ha}$ stands for Hartree energy) was taken, and an effective electron temperature of $0.04 \mathrm{eV}$ using a Fermi smearing function was used to help convergence.

A hexagonal unit cell was used to contain periodic cylindrical CNTs. A large $2 \mathrm{~nm}$ intertube spacing was used, which was sufficient to avoid interaction between neighbouring tubes, so that each tube could be considered as individual and free-standing. We modelled a collection of double-walled (DW), triple-walled (TW), and quadruple-walled (QW) zigzag and armchair nanotubes with diameters in the range of 1.65-5.50 nm (272-1056 atoms). In order to explore the intratube faceting between the tube shells in a DWCNT, the following combinations were investigated: $(n, 0) @(n+8,0),(n, 0) @(n+9,0)$, and $(n, 0) @(n+10,0)$ for zigzag helicity; and $(n, m) @(n+5, m+5)$ and $(n, m) @(n+6, m+6)$ for armchair helicity.

The Brillouin zone was sampled using a Monkhorst-Pack scheme. A $1 \times 1 \times 4$ $\Gamma$-centred k-point mesh was applied for structural optimisation. The initial nanotube geometry was symmetry-broken and then fully optimised by applying periodic boundary conditions. Structural optimisation convergence was reached when the change in position of every atom in each direction was less than $10^{-5} \mathrm{a}_{0}$ (where $\mathrm{a}_{0}$ is the Bohr radius) and the total energy converged to within a tolerance of $10^{-6} \mathrm{Ha}$. The optimised translational lattice vector was equal to $0.426 \mathrm{~nm}$, in excellent agreement with previous theoretical results obtained by hybrid DFT functional [26]. After relaxing the nanotube geometry, we doubled the unit cell length along the axial directions and added 3 iodine atoms, giving an average iodine-iodine spacing of $0.284 \mathrm{~nm}$ (comparable with the $0.3 \mathrm{~nm}$ experimentally measured 
by HRTEM imaging analysis for an encapsulated iodine atom chain in the literature [21,27]). We tested the iodine chains in two configurations: (i) adsorbed onto the innermost graphene shell surface, and (ii) intercalated between the graphene shells. All atoms were allowed to fully relax with no symmetry constraints, and iodine atoms were moved off-axis initially, allowing the breakage of linear symmetry.

We also analysed the existence of the iodine trimer both inside the internal graphene shell and intercalated between buckled graphene shells. The nanotube unit cell was repeated three times in this case, $\mathrm{C}$ atoms were fixed, and only the 3 iodine atoms were allowed to relax. $2 \mathrm{k}$-points were used along the tube direction. The charge transfer process for iodine encapsulated inside MWCNTs was determined by Mulliken population analysis, which has previously been successfully applied in the literature for iodine-nanocarbon interaction [28]. All calculations were performed without spin polarisation.

\section{Results and Discussion}

\subsection{TEM Data}

Figure 3 shows some examples of the HAADF images of the MWCNTs treated with iodine. Most often, the filling of the central channels with iodine (bright contrast) appeared quite limited, and only some scarce iodine clusters were present in the cavity (in the MWCNT on left hand side in Figure 3a, for instance). On the other hand, remarkably, iodine formed off-centred chainlike configurations (or lines, for simplicity) within the MWCNTs: one line and two lines for the left-hand and right-hand MWCNTs, respectively, can be seen in Figure 3a; three lines in Figure 3b; and four lines in Figure 3c. The enlarged view posted in the inset in Figure $3 b$ shows the dotted aspect of the lines, due to the fact that the imaging conditions were close to those of atomic resolution. The distances between the dots were $\sim 0.3 \mathrm{~nm}$, consistent with distances between iodine atoms within linear chains $[11,21,27]$. In contrast to the well-known filling of the central cavity of CNTs, the filling material (iodine) in this case appeared to be embedded within the MWCNT walls. Moreover, the iodine lines were systematically on only one side with respect to the longitudinal cross-section, thus filling the MWCNTs asymmetrically.
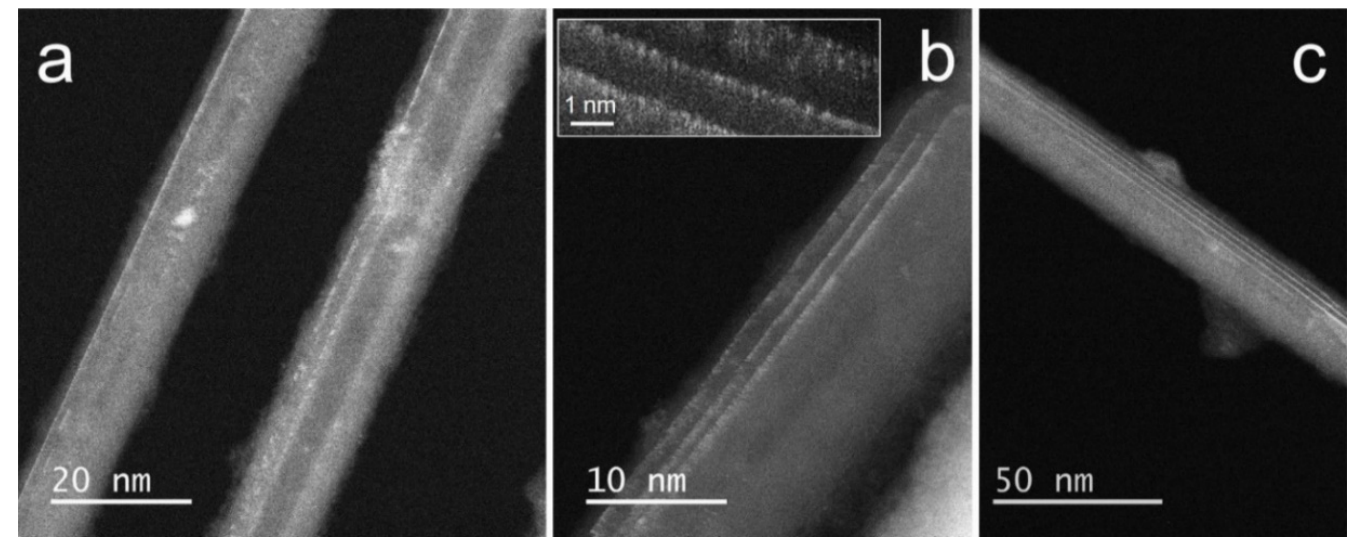

Figure 3. (a-c) HAADF images of arc-prepared MWCNTs treated with iodine. Bright contrast is due to the presence of iodine. Lower contrast is that of carbon only. The central cavity width of each MWCNT can be estimated thanks to its darker contrast. The inset in (b) is an enlarged detail from the image underneath, oriented differently.

Interestingly, in some cases, the iodine lines were found to follow an oblique path with respect to the longitudinal axis, suggesting that they were helically arranged (Figure $4 a, b$ ). 


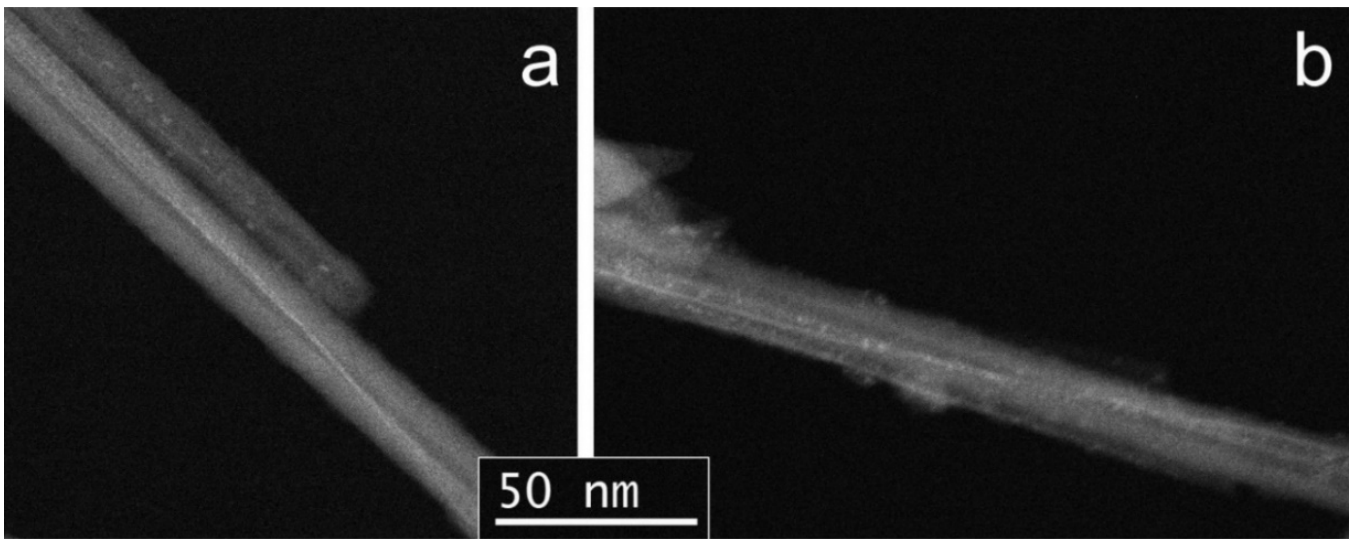

Figure 4. $(\mathbf{a}, \mathbf{b})$ HAADF images of arc-prepared MWCNTs treated with iodine showing examples of iodine lines probably following a helical path with respect to the MWCNT longitudinal axis.

When it was possible to count how many shells the MWCNTs contained (by observing the corresponding bright field images, as in Figure 5), it was observed that this asymmetric iodine-filling interfered with the shell counting.
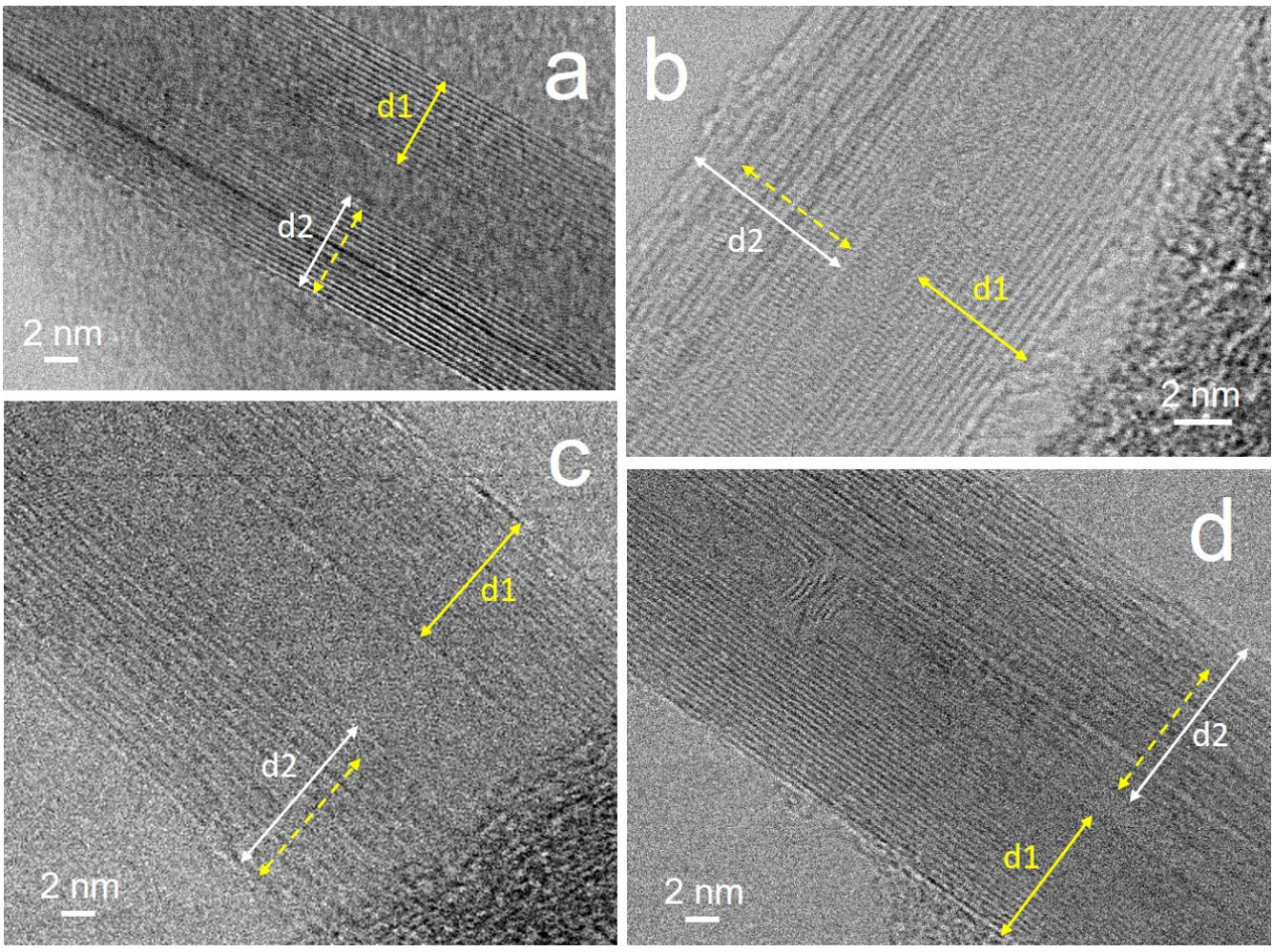

Figure 5. Examples of asymmetric thickness of the overall MWCNT walls: " $\mathrm{d} 2$ " is the thickness of the side of the MWCNT wall containing iodine line(s); "d1" is the thickness of the iodine-free side; dividing each $\mathrm{d} 1$ segment from the four images by the number of related intershell spacings provided average "regular" intershell distances ranging from 0.35 to $0.375 \mathrm{~nm}$; the dashed segment is a duplicate of the $\mathrm{d} 1$ segment put alongside $\mathrm{d} 2$ for better comparison of length differences. (a) The $\mathrm{d} 2$ side contained one iodine line (see the corresponding HAADF image in Supplementary Materials, Figure S1); (b) the d2 side contained 3 iodine lines (the corresponding HAADF image is Figure 3b); (c) iodine was present in the $\mathrm{d} 2$ side, but the number of iodine lines was hard to ascertain (see the corresponding HAADF image in Supplementary Materials, Figure S2); (d) the d2 side contained 4 iodine lines (the corresponding HAADF image is Figure $3 \mathrm{c}$ ). 
Considering the half-tube without iodine in Figure $5 b, d$, for instance, these MWCNTs had 14 and 24 shells, respectively. However, considering the half-tube containing iodine lines, they had 17 and 28 shells. The difference equaled the number of iodine lines observed for each MWCNT, i.e., 3 and 4, respectively. Correspondingly, the tube wall appeared thicker on the side containing the iodine lines, compared to the opposite side (Figure 5). Therefore, the lined-up iodine atoms, the consequent increase in the line count in the overall MWCNT wall, and the larger wall thickness on the MWCNT side containing iodine demonstrate that this one-sided, asymmetric filling was achieved by inserting iodine atoms between the concentric graphenes.

At this point, the question arises whether the iodine atoms are actually displayed as a single-atom-thick plane, as for regular intercalants, or as a single-atom-large linear chain, as for iodine when filling SWCNTs or DWCNTs [21]. Because of the oxidation treatment, it is clear that the intergraphene spacing had become accessible to iodine vapour (Figure 2). However, the spacing between the concentric graphenes $(0.35-0.375 \mathrm{~nm}$ on average, as calculated from Figure 5), governed by the van der Waals distance, was not large enough to allow iodine to intercalate, as an intergraphene distance of $\sim 0.73 \mathrm{~nm}$ would be needed [29]. The expansion of the intergraphene distance upon the intercalation of iodine was not possible either, due to the closed concentric configuration of the graphene cylinders. The only configuration that allows iodine or any intercalant to enter the intergraphene spacing in concentric MWCNTs with perfect graphenes without damaging the graphene lattice integrity is if the concentric display of the graphenes is actually scroll-like [30,31]. However, in that case, there would be no reason for the intercalation to take place on one side of the MWCNT wall only. A decisive demonstration for a linear, chainlike display of iodine atoms is shown in Figure 4, as the occurrence of oblique bright lines could not be possible if the lines were the trace of single-atom-thick, intercalated iodine layers seen edge-on. A consequence of this is that the space to be filled by iodine should have a nanosized, elongated channel morphology running parallel to the MWCNT axis.

Going back to the HRTEM investigation of the MWCNTs before iodine treatment (i.e., the oxidised MWCNTs) and the MWCNTs before the oxidation step (i.e., the asreceived MWCNTs), focusing on the MWCNT body instead of the MWCNT ends, a clear explanation is visible: anomalous, larger intergraphene distances are already present in the as-prepared MWCNTs, again one-sided (Figure 6). The anomalous distances were displayed randomly, and exhibited a variety of values; hence, they were not all suitable for iodine intercalation. Not all the MWCNTs exhibited this feature in the images, but since the anomalous intergraphene distances were one-sided, they required the MWCNTs to be properly oriented with respect to the electron beam to be revealed. Therefore, it cannot be said whether all the MWCNTs were affected. It is in any case clear that the one-sided filling by iodine was not due to the oxidation or the intercalation processes, but to a specific textural feature already present in the as-prepared MWCNTs. An asymmetry related to a disparity in the distribution of the intergraphene distances was already reported in the literature for arc-prepared MWCNTs [16-18], but was barely emphasised. It is also worth noting that such asymmetric features were never found in MWCNTs prepared by any process other than the electric arc (catalysed CVD, typically) investigated in our laboratory for decades, or reported in the literature, to the best of our knowledge.

Three potential causes can be found in the literature to explain asymmetrical features as seen in Figure 6 while fulfilling the requirement of having channel-like cavities parallel to the CNT axis.

The first was proposed by Liu et al. $[17,18]$. It requires considering the MWCNTs as polygonised, hence exhibiting cross-sections as sketched in Figure 7a: in the flat parts of the concentrically nested graphenes, the intergraphene distance $d$ is regular, whereas the intergraphene distance $h$ in the local region of high curvature is higher than $d$. This is unlikely because: (i) polygonised multigraphene shells which form in the same conditions along with the MWCNTs do not exhibit, with a similar occurrence frequency, such a difference in intergraphene spacing between the flat and the highly curved parts; 
(ii) even in cases where such a difference occurs, the extended intergraphene spacing barely reaches the $0.68 \mathrm{~nm}$ needed for iodine to intercalate; and (iii) the actual polygonisation of as-prepared arc-MWCNTs is questionable because, if it was a reality, HRTEM images should find the frequent occurrence of MWCNTs for which one side only exhibits graphene fringes, with the graphenes from the other side not being properly oriented with respect to the Bragg angle.

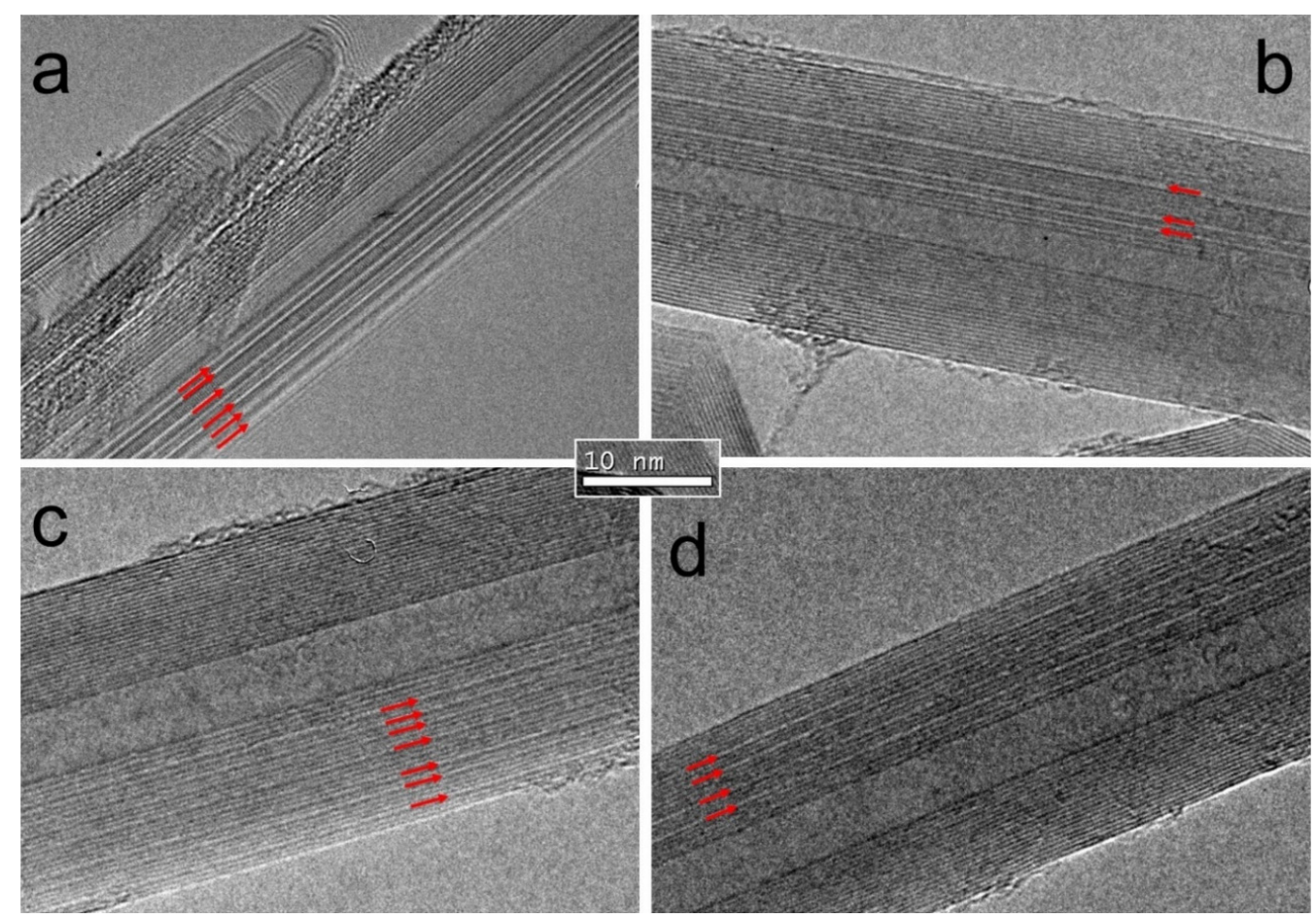

Figure 6. Examples of anomalous intergraphene distances (labelled with arrows) in the as-received, arc-prepared MWCNTs: $(\mathbf{a}, \mathbf{b})$ from Sigma Aldrich; $(\mathbf{c}, \mathbf{d})$ from MER Corporation. It is striking that they are systematically one-sided.

Another possible explanation was proposed in [19]. It requires one to consider the MWCNTs as no longer built following the nested (Russian doll) model but following a mix between this model and the scroll model, as sketched in Figure $7 \mathrm{~b}$, in a way that causes the same number of graphenes to appear on both sides of the central cavity when the image is formed with the TEM electron beam oriented as sketched. The main problems with this model are that: (i) the scroll-like model has never received direct experimental support but only indirect support based upon intercalation results which do not make the demonstration straightforward [32], and (ii) the growth scenario at the origin of Figure $7 \mathrm{~b}$ is highly conjectural. Overall, no reason is provided why the free graphene terminations should be all located on the same side of the MWCNTs.

Finally, a third configuration may be found in a serendipitous way in the work of Huang et al. [33]. In their simulations, they constructed MWCNTs with concentric texture and gave them intergraphene distances larger than the equilibrium distance related to the regular van der Waals interactions. When the structures were then relaxed using a van der Waals forcefield configured to the equilibrium intershell spacing, their calculations showed an asymmetric relaxation that brought the graphenes closer to each other, making the concentric MWCNTs adopt a droplet-like morphology including local decohesions forming nanochannels, all located and aligned on the same side of the MWCNT, within the bulge (Figure 7c). 


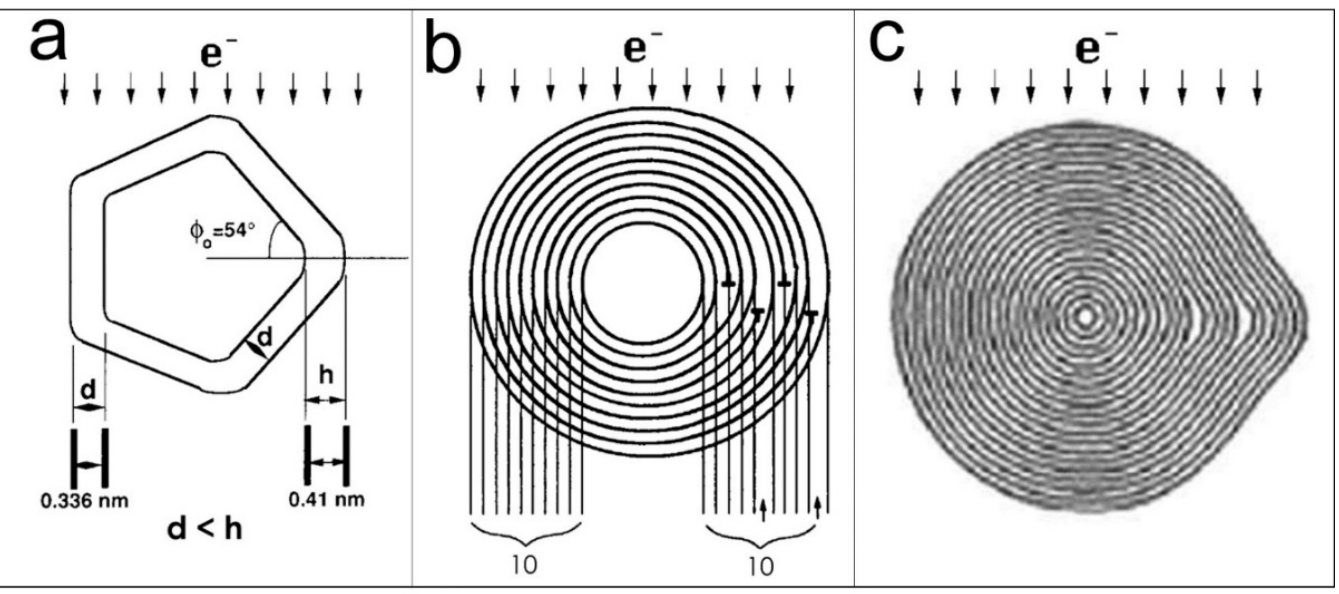

Figure 7. Sketches for three different MWCNT models potentially compatible with the observation of asymmetrically distributed anomalous intergraphene distances along with an even number of graphenes on both sides with respect to the central cavity, for TEM images taken with the electron

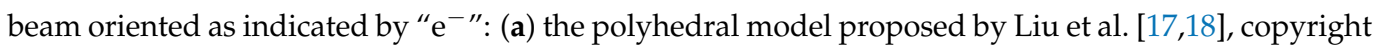
permission from Elsevier; (b) the combined concentric/scroll model proposed by Amelinckx et al. [19], copyright permission from AAAS; (c) a concentric model involving intergraphene distances larger than the equilibrium distance for van der Waals interactions, with subsequent asymmetric relaxation, as calculated by Huang et al. [33].

The latter model (Figure 7c) is far more convincing than the former two. This is because there is a simple and compulsory reason for the MWCNTs to be initially built with larger intergraphene distances than the equilibrium distance, namely, the effect of the anisotropy of the in-plane versus out-of-plane coefficient of thermal expansion (CTE) of graphene stacks (which can be taken to be equal to that of a graphite single crystal, as the structure type-graphitic or turbostratic - does not play a significant role [34]). The in-plane CTE of graphite is very limited, close to 0 on average [35], while the CTE perpendicular to the plane is fairly high, in the range of $30 \times 10^{-6} / \mathrm{K}$ or more (these CTE values are given as a range of order, as they appear to vary with temperature [34,35]). MWCNTs prepared by the electric arc process are formed at the contact of the arc cathode, whose surface can reach $5000{ }^{\circ} \mathrm{C}$ [4]. Thus, while the successive graphene cylinders are growing, they adopt the preferred intergraphene distance associated with a temperature of several thousand degrees. Upon cooling, the graphene cylinder diameters do not change much due to the low in-plane CTE, while the intergraphene distances shrink a lot down to the equilibrium distance because of the high CTE perpendicular to the plane. The MWCNTs are then in the same situation as those investigated in [33], and radially aligned longitudinal nanochannels are formed, distorting the MWCNT cross-section with a bulge. This mechanism is already documented, as, for instance, it was found to be responsible for the slit-like porosity present in the pyrolytic carbon interphase in carbon-fibre-reinforced ceramic matrix composites [36]. It also explains why the same longitudinal nanocavities cannot form in concentric MWCNTs prepared by methods other than the electric arc, because of the difference in the formation temperatures (in the range of $600-1000{ }^{\circ} \mathrm{C}$ for catalyst-enhanced CVD processes). Other causes play only a minor role, such as the number of graphene shells and the perfection of the graphenes involved, which are both usually lower in MWCNTs prepared by lowtemperature processes.

Figure 8 illustrates the effect of CTE, based on a plot proposed by Kellett et al. [34]. Using one of the plot lines related to pyrolytic carbon (presumably close to the behaviour of MWCNTs) and starting from the most frequent intergraphene distances in MWCNTs of any kind (0.332 to $0.335 \mathrm{~nm}$, according to [37]), the variation of the intergraphene distances in MWCNTs upon cooling is reported for both a synthesis temperature of $800^{\circ} \mathrm{C}$ (as a common temperature for CCVD processes) and $3500^{\circ} \mathrm{C}$ (as a plausible temperature during the arc 
process). The intergraphene distance shrinkage reaches only $2.7 \%$ for CCVD MWCNTs, whereas it is as high as $12.5 \%$ for arc MWCNTs.

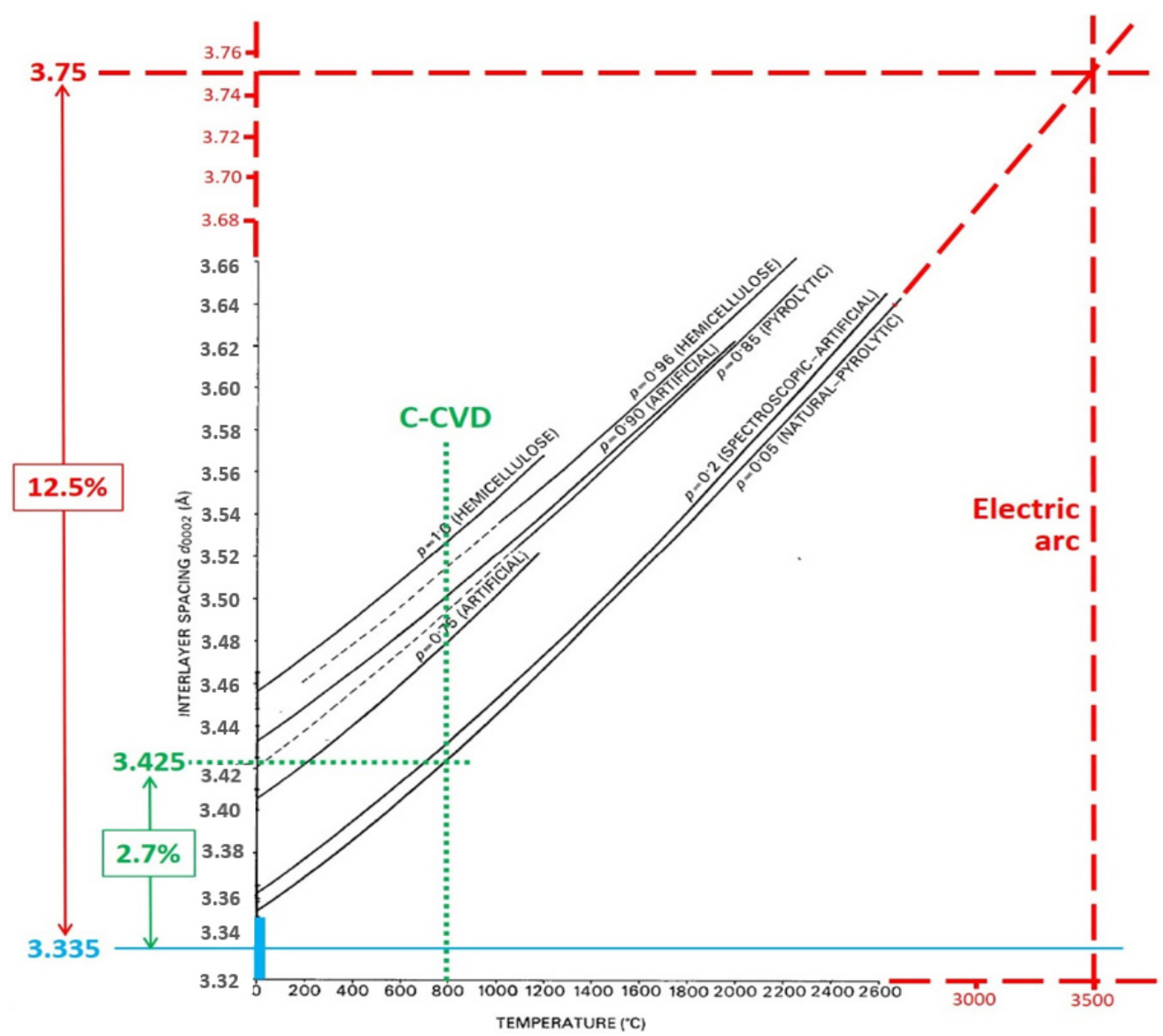

Figure 8. Plot of the variation of the intershell spacing (in Angström) with temperature for a variety of graphenic materials extensively modified from [34]. Several elements have been added to the original figure, in particular all the dotted and dashed lines, and the related labels (coloured in the online version). The thick blue segment at the bottom of the $y$ axis represents the range of most frequent intergraphene spacings in MWCNTs at room temperature, regardless of the synthesis procedure [37], from which we considered the median value $(0.3335 \mathrm{~nm})$. Dashed lines (in red) and related labels relate to the electric arc synthesis; dotted lines (in green) and related labels relate to the CCVD synthesis.

Returning to the observation of oblique iodine lines as illustrated by Figure 4, a hypothesis consistent with the model of Figure 7c is that some of the individual graphene cylinders making up the MWCNT wall may present an intrinsic, natural torsion, provided that they exhibit a helical structure, making them chiral [38]. When constructing a MWCNT, such a torsioned graphene cylinder has its CTE components rotated by a small angle with respect to the longitudinal axis of the CNT. Thus, upon cooling and subsequent relaxation, the nanocavity formed would also follow that torsion, explaining the oblique iodine line that fills it.

\subsection{Modelling}

We next explored this CTE-based mechanism further with DFT calculations. The temperature-dependency expansion of the $\mathrm{C}-\mathrm{C}$ bond length is not included in the current calculations, since, as discussed above, the in-plane lattice variation from 300 to $3500{ }^{\circ} \mathrm{C}$ is only of $7 \times 10^{-4} \mathrm{~nm}$ [35]. We first explored the cross-sectional variation induced by varying the intershell spacing, via different DWCNT zigzag shell combinations (Figure 9). 


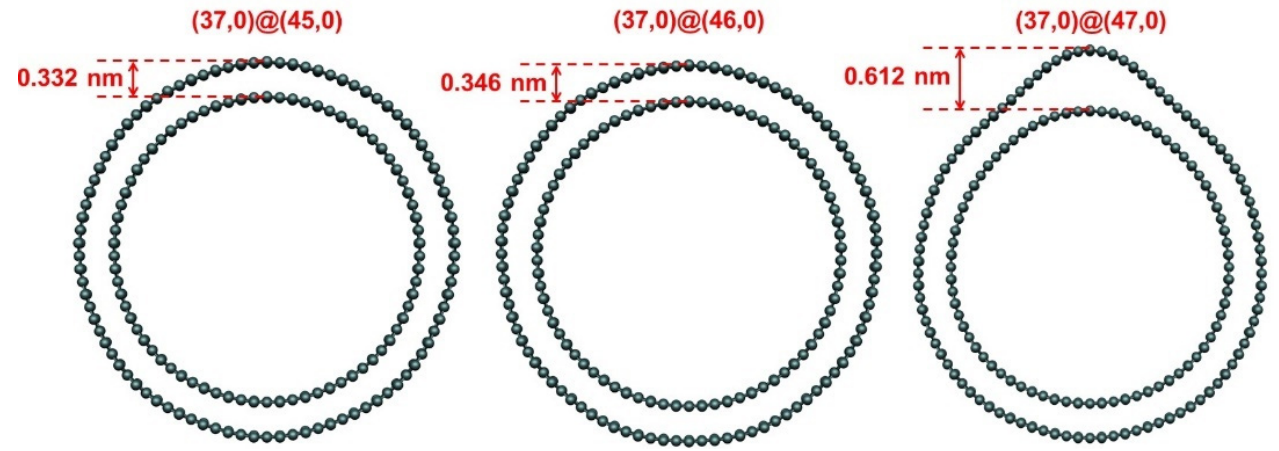

Figure 9. DFT-D2 relaxed cross-sections for different helicity combinations of zigzag DWCNTs. Intershell spacings are marked in $\mathrm{nm}$. Note that the structure on the right is asymmetric with buckling deformation, hence forming a channel-like cavity (the intershell spacing in the DWCNT part opposed to the bulge is $\sim 0.344 \mathrm{~nm}$ ).

For the shell combinations $(n, 0) @(n+10,0)$, the intershell distance was initially equal to $0.376 \mathrm{~nm}$ for the two nonrelaxed cylinders, which nearly matched the intershell spacing value found for arc-MWCNTs at $3500{ }^{\circ} \mathrm{C}$. After off-axis nonsymmetric geometrical optimisation, the tubular configuration changed and we observed a buckling deformation along one longitudinal side with an intershell spacing of $0.618-0.628 \mathrm{~nm}$. The intershell spacing in the nonbuckled side $(0.342-0.347 \mathrm{~nm})$ closely matched that of the turbostratic structure $(0.344 \mathrm{~nm})$. All the geometry parameters are summarised in Table 1.

Table 1. DFT-D2 optimised geometry parameters of $(n, 0) @(n+x, 0)$ zigzag DWCNT combinations, where $x=8,9$, and 10. The intershell spacing $d$ of the unrelaxed case when both shells had the same central axis are indicated in brackets beneath the DWCNT description. The minimum $\left(d_{\min }\right)$ and maximum $\left(d_{\max }\right)$ intershell distance and the difference between them $(\Delta d)$ are given in $\mathrm{nm}$. Only some of the points reported in Figure 10 are listed here as representative points. The whole data set is provided in Supplementary Materials.

\begin{tabular}{|c|c|c|c|c|}
\hline Tube Combination ( $\left.d_{\text {unrelaxed }}\right)$ & Helicity Index & $d_{\min }(\mathrm{nm})$ & $d_{\max }(\mathrm{nm})$ & $\Delta d(\mathrm{~nm})$ \\
\hline \multirow{5}{*}{$\begin{array}{l}(n, 0) @(n+8,0) \\
(0.313 \mathrm{~nm})\end{array}$} & $(13,0) @(21,0)$ & 0.319 & 0.319 & 0.000 \\
\hline & $(25,0) @(33,0)$ & 0.326 & 0.326 & 0.000 \\
\hline & $(37,0) @(45,0)$ & 0.332 & 0.332 & 0.000 \\
\hline & $(49,0) @(57,0)$ & 0.323 & 0.335 & 0.013 \\
\hline & $(61,0) @(69,0)$ & 0.325 & 0.336 & 0.011 \\
\hline \multirow{5}{*}{$\begin{array}{l}(n, 0) @(n+9,0) \\
(0.344 \mathrm{~nm})\end{array}$} & $(13,0) @(22,0)$ & 0.349 & 0.355 & 0.006 \\
\hline & $(25,0) @(34,0)$ & 0.345 & 0.347 & 0.002 \\
\hline & $(37,0) @(46,0)$ & 0.343 & 0.346 & 0.003 \\
\hline & $(49,0) @(58,0)$ & 0.336 & 0.346 & 0.010 \\
\hline & $(61,0) @(70,0)$ & 0.336 & 0.347 & 0.011 \\
\hline \multirow{5}{*}{$\begin{array}{c}(n, 0) @(n+10,0) \\
(0.376 \mathrm{~nm})\end{array}$} & $(13,0) @(23,0)$ & 0.343 & 0.568 & 0.225 \\
\hline & $(25,0) @(35,0)$ & 0.342 & 0.618 & 0.276 \\
\hline & $(37,0) @(47,0)$ & 0.347 & 0.612 & 0.265 \\
\hline & $(49,0) @(59,0)$ & 0.346 & 0.628 & 0.282 \\
\hline & $(61,0) @(71,0)$ & 0.345 & 0.625 & 0.280 \\
\hline
\end{tabular}

The comparison of the minimum and maximum intershell spacing of the relaxed structures ( $\Delta d$ in Table 1 ) showed three regimes. When $\Delta d$ was in the range $0.000-0.004 \mathrm{~nm}$, this indicated that both the inner and outer shells had essentially uniform circular cross- 
sections. This was the case for the DWCNTs with smaller intershell spacings and smaller diameters. In the range $\Delta d=0.010-0.015 \mathrm{~nm}$, the CNTs were becoming polygonal and the intershell spacing was therefore becoming more variable. This was observed for tubes with larger diameters. The third regime, where $\Delta d=0.225-0.288 \mathrm{~nm}$, corresponded to the buckling regime, where cross-sectional distortion became highly localised.

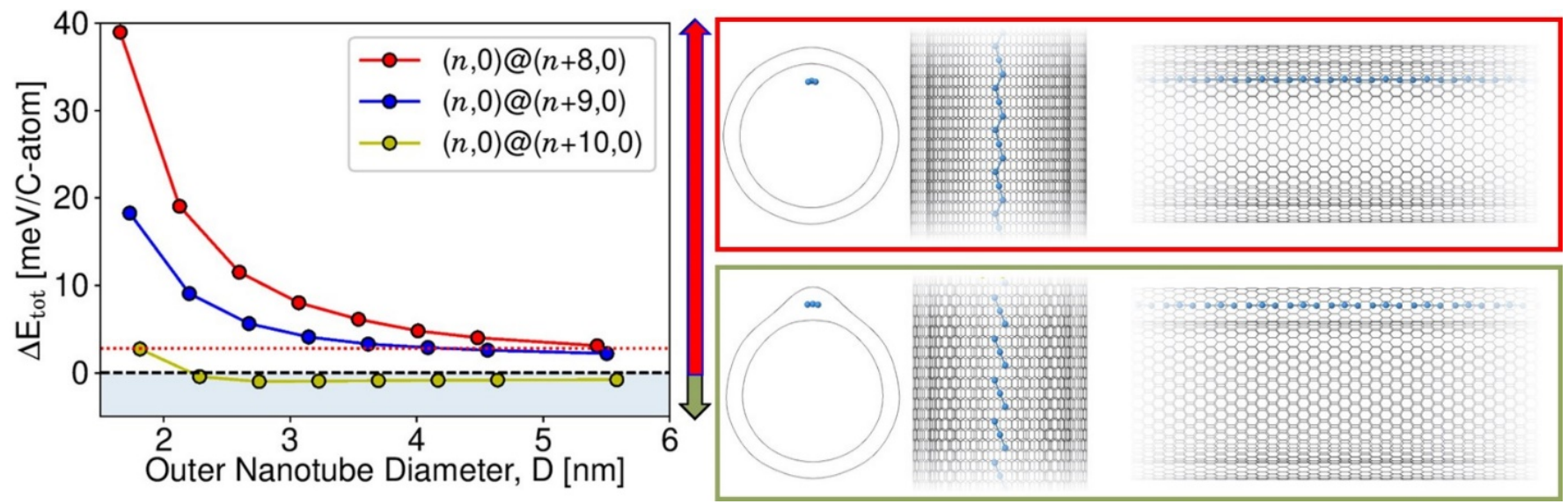

Figure 10. Left: DFT-D2 total energy difference per carbon atom (in meV/C-atom) between iodine intercalated within the graphene shells and iodine adsorbed onto the innermost shell surface, for zigzag DWCNTs. Three families of DWCNTs were considered: $(n, 0) @(n+8,0)($ red $),(n, 0) @(n+9,0)($ blue), and $(n, 0) @(n+10,0)$ (green) for different diameters. The x-axis uses the diameter of the equivalent circular external shell. The shaded lower section indicates shell combinations in DWCNTs for which the intercalation of iodine between the shells was favoured. DWCNTs with $\Delta E_{t o t}>0$ were stabilised with the iodine chain adsorbed onto the innermost shell surface (red arrow). DWCNTs with $\Delta E_{\text {tot }}<0$ were energetically favoured when the iodine chain was intercalated into the nanocavity on the buckled side (green arrow). The horizontal red dotted line represents the energy difference per carbon atom for an AA-stacked bilayer graphene (2LG) between the case where iodine was confined in the intergraphene space and the case where iodine was adsorbed on the 2LG surface. Right: Optimised cross-sections and top and side views of $\mathrm{I}(49,0) @(57,0)$ (red frame) and I@(49,0)@(59, 0) (green frame) DWCNTs.

To establish when iodine intercalation is an exo- or endothermic process, we compared the energetics of the DWCNTs when an iodine chain was confined either inside the inner shell or between the nanotube shells, as follows:

$$
\Delta E_{t o t}=\frac{1}{N_{C}}\left(E_{t o t}^{\text {inter }}-E_{\text {tot }}^{\text {inside }}\right)
$$

where $E_{\text {tot }}^{\text {inter }}$ is the total energy of I@DWCNT with iodine intercalated between nanotube shells, $E_{\text {tot }}^{\text {inside }}$ is the total energy of I@DWCNT with iodine inside the internal shell, and $N_{C}$ is the number of carbon atoms within the simulation cell.

Figure 10 shows the total energy difference $\left(\Delta E_{t o t}\right)$ per carbon atom between the innermost shell and the intershell configurations for the graphene-shell helicity combinations mentioned above, over a range of diameters.

For the $(n, 0) @(n+8,0)$ and the $(n, 0) @(n+9,0)$ combinations, the iodine chain could not be stabilised between the CNT shells and was only stable if confined inside the smaller one. The iodine chain preferred to stick to the CNT inner surface, mainly due to $\mathrm{vdW}$ interactions [28]. The trend of decreasing binding with diameter was due to the emergence of polygonisation, which occurs for large-diameter CNTs [39]. The vertices of the carbon polygons introduced a local out-of-plane stress, leading to an increase in the interlayer spacing from $0.325 \mathrm{~nm}$ to $0.336 \mathrm{~nm}$. This helped to stabilise the iodine species in intrashell position, even if this configuration still remained unstable compared to iodine in 
the central cavity. For the $(n, 0) @(n+10,0)$ concentric graphene shell pairs, the intershell iodine filling became energetically more favourable. The only exception was the smallest diameter DWCNT, but in this case the tubes were so small that localised buckling could not occur and the tube underwent elliptical distortion instead. This DWCNT had a smaller internal shell diameter than any that have been seen experimentally and hence is only of theoretical interest.

Upon adding iodine, the intershell spacing in the buckle increased to $0.688 \mathrm{~nm}$, in excellent agreement with the direct measurement of $\sim 0.69 \mathrm{~nm}$, as obtained in three instances from the HRTEM images showing pairs of graphene fringes intercalated with an iodine line reported in Figure 5.

Thus, the distance between MWCNT shells containing intercalated iodine could be lower with respect to the experimental value recorded for graphite co-intercalated with iodine and bromine (a smaller ion) in the literature $(\sim 0.73 \mathrm{~nm})$ [29]. Iodine is more stable inside the buckle than when inside the CNT cavity due to the stronger charge transfer when fully confined (see Table 2).

Table 2. DFT-D2 computed charge transfer (electrons per iodine atom) for inside and intercalated configuration for iodine in zigzag $(n, 0) @(n+10,0)$ DWCNTs. Negative number indicates charge transfer from the tube to the iodine atom.

\begin{tabular}{cccc}
\hline DWCNT Type & Inside & Intercalated & Charge Transfer Difference \\
\hline$(13,0) @(23,0)$ & -0.25 & -0.19 & +0.06 \\
\hline$(19,0) @(29,0)$ & -0.20 & -0.26 & -0.06 \\
\hline$(25,0) @(35,0)$ & -0.19 & -0.27 & -0.09 \\
\hline$(31,0) @(41,0)$ & -0.18 & -0.27 & -0.09 \\
\hline$(37,0) @(47,0)$ & -0.18 & -0.27 & -0.09 \\
\hline$(43,0) @(53,0)$ & -0.18 & -0.28 & -0.10 \\
\hline$(49,0) @(59,0)$ & -0.17 & -0.28 & -0.11 \\
\hline$(61,0) @(0,71)$ & -0.17 & -0.28 & -0.11 \\
\hline
\end{tabular}

The same analysis was next applied to infinite flat bilayer graphene (2LG) with various stacking. Like the $(n, 0) @(n+8,0)$ and $(n, 0) @(n+9,0)$ DWCNTs, iodine intercalation was not energetically favoured with respect to iodine adsorbed on the 2LG surface by $3.09 \mathrm{meV} / \mathrm{C}$-atom (red dotted line in Figure 10; see also Supplementary Materials, Figures S3 and S4, and related comments). The energy difference for the largest-diameter $(n, 0) @(n+8,0)$ DWCNT approached that of the AA-stacking bilayer graphene value, as a logical consequence of the distance between the graphenes in the iodine-filled bulge part $(0.655 \mathrm{~nm})$ for the DWCNT which was almost identical with that obtained for the iodine-intercalated 2LG $(0.659 \mathrm{~nm})$. This was consistent with the literature dealing with the intercalation of iodine in graphemic carbons, where iodine has been seen to fill CNT cavities and inter-CNT spacings in bundle but does not intercalate into graphite where the interlayer spacing is too small $[11,26,40]$, and further reinforced the importance of the local buckling in the present case for mediating the intercalation process. The ground-state configuration for the chain in the $(n, 0) @(n+10,0)$ tube involved a small degree of symmetry breaking with the iodine atoms slightly nonlinear, grouping instead into compressed $\mathrm{I}_{3}$ units with $0.292 \mathrm{~nm}$ iodine bonds. The resulting charge transfer was 0.27 e per iodine atom, consistent with previous published data for iodine encapsulated in narrow CNTs [11,28].

This precise dependence on the intershell spacing was remarkably independent of other system parameters. Calculations on armchair CNTs (see Supplementary Materials, Tables S2 and S3) showed the same buckling effect, i.e., the buckling and iodine filling is purely a function of the intershell spacing and is independent of helicity. The same energetic preference was seen for tri-iodide as iodine chains (see Supplementary Materials, Figure S5). The averaged Mulliken charge transfer for the tri-iodide molecules intercalated 
between buckled walls was 0.34 e per iodine atom, matching the experimental integer value -1 for $\mathrm{I}_{3}^{-}$anion reported in the literature [28]. The double unit cell value of $0.27 e$ was slightly lower because of the additional Coulombic confinement [25]. The calculations also showed the same behaviour with both three- and four-wall CNTs (see Supplementary Materials, Figures S6-S9). The stabilisation energy of iodine intercalated within a buckle could be fitted by an exponential, linking it to the distance of the iodine from the nanotube axis. This function tended to $383 \mathrm{meV} / \mathrm{I}$-atom as the tube size increased (see Supplementary Materials, Figure S8). A precise quantitative picture of the behaviour of an iodine chain in these systems would require modelling longer supercells [28] due to the low symmetry matching between the unit cell length and the preferred interiodine spacing. Nonetheless, the key result here is the comparative behaviour between the 'inner surface adsorbed' and 'intercalated' cases, which was qualitatively independent of the cell sizes chosen. This was tested by comparing our (double unit cell +3 iodine atoms) systems to (single unit cell +1 iodine atom) systems (not shown); the same qualitative behaviour as presented here was found.

\section{Conclusions}

An attempt to fill oxidised arc-MWCNTs with iodine by a known procedure ended with iodine lines occupying intershell spaces on one side only (with respect to the longitudinal axis) of the MWCNTs. When comparing the iodine-free and iodine-filled sides, the latter were always thicker. This configuration was only possible with the existence of one-sided nanocavities (wide enough to accommodate iodine species) between the shells. The distribution of the iodine lines (and hence, of the nanocavities) originated from the random and one-sided occurrence of anomalous intershell spacing observed in pristine (as-prepared) arc-MWCNTs. Thanks to the large difference between the in-plane and out-of-plane components of the coefficient of thermal expansion, this distortion and the subsequent formation of the off-axis elongated nanochannels occurred after anisotropic thermal relaxation, due to the shrinking of the graphene cylinder diameters when cooling from the very high processing temperatures $\left(\sim 3500{ }^{\circ} \mathrm{C}\right)$ down to room temperature. Because processing temperatures for MWCNT synthesis via procedures other than the electric arc method are much lower (typically $<1000{ }^{\circ} \mathrm{C}$ ), this anomalous feature was assumed to be specific to arc-MWCNTs. DFT calculations confirmed that the iodine intercalation between the shells was only exothermic when such buckling was present, independently of the chirality, iodine arrangement (chains or molecules), or neighbouring walls. Since these longitudinal edge cavities are universal in arc-MWCNTs, it is possible that they may also be filled by other species such as nitrogen gas, water, or linear chains of small metal atoms.

Supplementary Materials: The following supporting information can be downloaded at: https: / / www.mdpi.com/article/10.3390/c8010010/s1. Figure S1: low magnification HAADF image corresponding to the bright-field image shown in Figure 5a of the main text; Figure S2: low magnification HAADF image corresponding to the bright-field image shown in Figure $5 \mathrm{c}$ of the main text; Figure S3: schematic representation of the displacement along the zig-zag direction (horizontal black arrow) of the lattice structures of two superimposed graphenes forming a 2LG. The lattice of the lower fixed layer is represented in black, while that of the moving upper one is represented in red and blue for the AA and AA' stacking systems, respectively [41-43]; Figure S4: DFT-D2 computed total energy difference between iodine either intercalated or adsorbed for (red) AB'- and (blue) AA-stacked 2LG as a function of the layer width. Below is provided a model of the bilayer graphene showing the formation of the buckled bulb after iodine intercalation that has been relaxed. Dashed red rectangle represents the conventional unit cell $\left(6.171 \mathrm{~nm}\right.$ in this case). It is widely accepted that $\mathrm{AA}^{\prime}$-stacked bilayer is energetically favoured compared to AA-stacked configuration. However, interestingly, iodine intercalation changes this hierarchy, making the AA-stacking the lowest energy one [44,45]; Figure S5: side view of DFT-D2 relaxed super-cell of tri-iodide-filled $(25,0) @(35,0)$ zig-zag DWCNT. Two positions for the iodine trimer were tested: (left) intercalated between the graphene shells, and (right) inside the internal shell. The intercalated structure on the left is more stable, by $+0.720 \mathrm{meV}$ per carbon atom (+172.933 meV per iodine atom); Figure S6: optimised structure of iodine-filled (top) 
$(25,0) @(33,0) @(43,0)$ and (bottom) $(25,0) @(35,0) @(43,0)$ zig-zag TWCNTs. Two positions for the iodine line were tested: (left) intercalated between shells with the helical index difference set to 10; (right) inside the innermost shell. All energy values (in meV/C-atom) are given with respect to the most stable configurations shown on the left; Figure S7: optimised structure of iodine-filled (top) $(37,0) @(45,0) @(55,0)$ and (bottom) $(37,0) @(47,0) @(55,0)$ zig-zag TWCNTs. Two positions for the iodine line were tested: (left) intercalated between shells with the helical index difference set to 10, (right) inside the innermost shell. All energy values (in meV/C-atom) are given with respect to the most stable configurations placed on the left; Figure S8: DFT-D2 calculated total energy difference per iodine atom of (green circles) DWCNTs and (magenta triangles) TWCNTs versus the separation distance between iodine atom and nanotube axis. The solid black curve shows the exponential fitting equation: $\Delta E_{\text {tot }}(\mathrm{meV} / \mathrm{I}$-atom $)=-383+8435 \exp (-3.67 d)$, where $d$ represents the separation distance between the iodine line and the nanotube centre; Figure S9: optimised structure of iodine-filled $(25,0) @(33,0) @(43,0) @(51,0)$ zig-zag QWCNT. Two positions for iodine line were tested: (left) intercalated between shells with the helical index dif-ference set to 10, (right) inside the innermost shell. All energy values (in meV/C-atom) are given with respect to the most stable configuration shown on the left; Table S1: DFT-D2 optimised geometry parameters of zig-zag DWCNT $(n, 0) @(n+x, 0)$ combinations, where $x=8,9$, and 10. The intershell spacing $\mathrm{d}$ of the unrelaxed case when both shells have the same central axis are indicated in brackets beneath the tube description. The minimum $\left(d_{\text {min }}\right)$ and maximum $\left(d_{\text {Max }}\right)$ intershell distance, and the difference between them $(\Delta d)$ are given in $\mathrm{nm}$. This table includes all values represented in Figure 10 shown in the main text; Table S2: DFT-D2 optimised geometry parameters of armchair DWCNT $(n, n) @(n+x, n+x)$ combinations, where $x=5$ and 6 . The diameter of the outer shell (in $\mathrm{nm}$ ) is provided, as well the minimum $\left(d_{\min }\right)$ and the maximum $\left(d_{M a x}\right)$ intershell distance between the un-buckled and the buckled sides, respectively. $\Delta d$ is the difference between these two values. Bracketed numbers in the left column indicate the intershell spacing for the initial unrelaxed axially symmetric cylindrical tubes; Table S3: calculated DFT-PBE+D2 energy difference (meV/C-atom) between a linear iodine chain inside the internal shell and the same intercalated between the carbon shells for various zig-zag and armchair DWCNTs. A negative energy difference indicates the iodine is more stable when intercalated between the carbon shells.

Author Contributions: Conceptualization, A.C.T.-D., M.M. and C.E.; methodology, A.C.T.-D., A.I., E.P., M.M., C.E. and A.P.; software, A.I. and C.E.; investigation, A.C.T.-D., A.I., L.N., E.P., C.E. and M.M.; writing-original draft preparation, A.C.T.-D., M.M. and A.I.; writing-review and editing, all the authors; supervision, M.M., C.E. and A.P.; project administration, C.E., M.M. and A.P. All authors have read and agreed to the published version of the manuscript.

Funding: This research was funded by the 'EdgeFiller' project (grant \# ANR-16-CE24-0008-02) and the 'OPIFCat' project (grant \# ANR-20-CE08-0026).

Institutional Review Board Statement: Not applicable.

Informed Consent Statement: Not applicable.

Data Availability Statement: Data are contained within the article and the Supplementary Materials document.

Acknowledgments: We thank the CCIPL (Centre de Calculs Intensifs des Pays de la Loire), where many of the calculations were performed. A. Descamps-Medine (UMS Castaing, Toulouse) is thanked for operating the Jeol ARM microscope, and P. Puech (CEMES, Toulouse) for fruitful discussion.

Conflicts of Interest: The authors declare no conflict of interest.

\section{References}

1. Singh, D.K.; Iyer, P.K.; Giri, P.K. Diameter dependence of interwall separation and strain in multiwalled carbon nanotubes probed by X-ray diffraction and Raman scattering studies. Dia. Relat. Mater. 2010, 19, 1281-1288. [CrossRef]

2. Iijima, S. Helical microtubules of graphitic carbon. Nature 1991, 354, 56-58. [CrossRef]

3. Monthioux, M.; Kuznetsov, V.L. Who should be given the credit for the discovery of carbon nanotubes? Carbon 2006, 44, 1621-1623. [CrossRef]

4. Monthioux, M.; Serp, P.; Caussat, B.; Flahaut, E.; Razafinimanana, M.; Valensi, F.; Laurent, C.; Peigney, A.; Mesguich, D.; Weibel, A.; et al Carbon nanotubes. In Handbook of Nanotechnology, 4th ed.; Bhushan, B., Ed.; Springer: Heidelberg, Germany, 2017 ; pp. $193-247$.

5. Monthioux, M. Filling single-wall carbon nanotubes. Carbon 2002, 40, 1809-1823. [CrossRef] 
6. Monthioux, M.; Flahaut, E.; Cleuziou, J.-P. Hybrid carbon nanotubes: Strategy, progress, and perspectives. J. Mater. Res. 2006, 21, 2774-2793. [CrossRef]

7. Bousige, C.; Stolz, A.; Silva-Santos, S.D.; Shi, J.; Cui, W.; Nie, C.; Marques, M.A.L.; Flahaut, E.; Monthioux, M.; San-Miguel, A Superior carbon nanotube stability by molecular filling: A single-chirality study at extreme pressures. Carbon 2021, 183, 884-892. [CrossRef]

8. Kim, S.H.; Choi, W.I.; Kim, G.; Song, Y.J.; Jeong, G.-H.; Hatakeyama, R.; Ihm, J.; Kuk, Y. Cesium-filled single wall carbon nanotubes as conducting nanowires: Scanning tunneling spectroscopy study. Phys. Rev. Lett. 2007, 99, 256407. [CrossRef]

9. Wang, Z.; Shi, Z.; Gu, Z. Synthesis of single-walled carbon nanotube/metal nanoparticle hybrid materials from potassium-filled nanotubes. Carbon 2010, 48, 443-446. [CrossRef]

10. Rao, A.M.; Eklund, P.C.; Bandow, S.; Thess, A.; Smalley, R.E. Evidence for charge transfer in doped carbon nanotube bundles from Raman scattering. Nature 1997, 388, 257-259. [CrossRef]

11. Zubaïr, A.; Tristant, D.; Nie, C.; Tsentalovitch, D.E.; Headrick, R.J.; Pasquali, M.; Kono, J.; Meunier, V.; Flahaut, E.; Monthioux, M.; et al. Charged iodide in chains behind the highly efficient iodine doping in carbon nanotubes. Phys. Rev. Mater. 2017, 1, 064002. [CrossRef]

12. Rossella, F.; Soldano, C.; Onorato, P.; Bellani, V. Tuning electronic transport in cobalt-filled carbon nanotubes using magnetic fields. Nanoscale 2014, 6, 788-794. [CrossRef] [PubMed]

13. Borowiak-Palen, E.; Mendoza, E.; Bachmatiuk, A.; Rummeli, M.H.; Gemming, T.; Nogues, J.; Skumryev, V.; Kalenczuk, R.J.; Pichler, T.; Silva, S.R.P. Iron filled single-wall carbon nanotubes-A novel ferromagnetic medium. Chem. Phys. Lett. 2006, 421, 129-133. [CrossRef]

14. Monthioux, M. (Ed.) Carbon Meta-Nanotubes: Synthesis, Properties, and Applications; Wiley-Blackwell: Chichester, UK, 2012.

15. Chopra, N.G.; Benedict, L.X.; Crespi, V.H.; Cohen, M.L.; Louie, S.G.; Zettl, A. Fully collapsed carbon nanotubes. Nature 1995, $377,135-138$. [CrossRef]

16. Li, Z.G.; Fagan, P.J.; Liang, L. The three-dimensional shape of carbon nanotubes by high-resolution electron microscopy. Chem. Phys. Lett. 1993, 207, 148-152. [CrossRef]

17. Liu, M.; Cowley, J.M. Structures of carbon nanotubes studied by HRTEM and nanodiffraction. Ultramicroscopy 1994, 53, 333-342. [CrossRef]

18. Liu, M.; Cowley, J.M. Structures of the helical carbon nanotubes. Carbon 1994, 32, 393-403. [CrossRef]

19. Amelinckx, S.; Bernaerts, D.; Zhang, X.B.; Van Tendeloo, G.; Van Landuyt, J. A structure model and growth mechanism for multishell carbon nanotubes. Science 1995, 267, 1334-1338. [CrossRef]

20. Nie, C.; Galibert, A.-M.; Soula, B.; Flahaut, E.; Sloan, J.; Monthioux, M. A new insight on the mechanisms of filling closed carbon nanotubes with molten metal iodides. Carbon 2016, 110, 48-50. [CrossRef]

21. Nie, C.; Galibert, A.-M.; Soula, B.; Sloan, J.; Flahaut, E.; Monthioux, M. The unexpected complexity of filling double-wall carbon nanotubes with nickel (and iodine) 1D nanocrystals. IEEE Trans. Nanotechnol. 2017, 16, 759-766. [CrossRef]

22. Rayson, M.J.; Briddon, P.R. Rapide iterative method for electronic-structure eigenproblems using localised basis functions. Comput. Phys. Commun. 2008, 178, 128-134. [CrossRef]

23. Rayson, M.J.; Briddon, P.R. Highly efficient method for Kohn-Sham density functional calculations of 500- atom systems Phys. Rev. B 2009, 80,1-11. [CrossRef]

24. Rayson, M.J. Rapid filtration algorithm to construct a minimal basis on the fly from a primitive Gaussian basis. Comput. Phys. Commun 2010, 181, 1051-1056. [CrossRef]

25. Grimme, S. Semiempirical GGA-type density functional constructed with a long-range dispersion correction. J. Comput. Chem. 2006, 27, 1787-1799. [CrossRef] [PubMed]

26. Komsa, H.-P.; Senga, R.; Suenaga, K.; Krasheninnikov, A.V. Structural Distortions and charge density waves in iodine chains encapsulated inside carbon nanotubes. Nano Lett. 2017, 17, 3694-3700. [CrossRef]

27. Chorro, M.; Kané, G.; Alvarez, L.; Cambedouzou, J.; Paineau, E.; Rossberg, A.; Kociak, M.; Aznar, R.; Pascarelli, S.; Launois, P.; et al. 1D-confinement of polyiodides inside single-wall carbon nanotubes. Carbon 2013, 52, 100-108. [CrossRef]

28. Rybkovskiy, D.V.; Impellizzeri, A.; Obraztsova, E.D.; Ewels, C.P. Polyiodide structures in thin single-walled carbon nanotubes: A large-scale density-functional study. Carbon 2019, 142, 123-130. [CrossRef]

29. Hung, C.-C.; Kucera, D. Graphite intercalation compound with iodine as the major intercalate. Carbon 1994, 32, 1441-1448. [CrossRef]

30. Mordkovich, V.Z. Three types of behavior of multiwall carbon nanotubes in reactions with intercalating agents. Mol. Cryst. Liq. Cryst. 2000, 340, 775-780. [CrossRef]

31. Duclaux, L. Review of the doping of carbon nanotubes (multiwalled and single-walled). Carbon 2002, 40, 1751-1764. [CrossRef]

32. Mordkovich, V.Z.; Baxendale, M.; Yoshimura, S.; Chang, R.P.H. Intercalation into carbon nanotubes. Carbon 1996, $34,1301-1303$. [CrossRef]

33. Huang, X.; Liang, W.; Zhang, S. Radial corrugations of multi-walled carbon nanotubes driven by inter-wall nonbonding interactions. Nanosc. Res. Lett. 2011, 6, 53. [CrossRef] [PubMed]

34. Kellett, E.A.; Richards, B.P. The c-axis thermal expansion of carbons and graphites. J. Appl. Cryst. 1971, 4, 1-8. [CrossRef]

35. Kellett, E.A.; Richards, B.P. The thermal expansion of graphite within the layer plane. J. Nucl. Mater. 1964, 12, 184-192. [CrossRef]

36. Després, J.-F.; Monthioux, M. Mechanical properties of C/SiC composites as explained from their interfacial features. J. Europ. Ceram. Soc. 1995, 15, 209-224. [CrossRef] 
37. Kharissova, O.V.; Kharisov, B.I. Variations of interlayer spacing in carbon nanotubes. RSC Adv. 2014, 4, 30807-30815. [CrossRef]

38. Vercosa, D.G.; Barros, E.B.; Souza Filho, A.G.; Mendes Filho, J.; Samsonidze, G.G.; Saito, R.; Dresselhaus, M.S. Torsional instability of chiral carbon nanotubes. Phys. Rev. B 2010,81, 165430. [CrossRef]

39. Leven, I.; Guerra, R.; Vanossi, A.; Tosatti, E.; Hod, O. Multiwalled nanotube faceting unravelled. Nat. Nanotechnol. 2016, 11, 1082-1086. [CrossRef]

40. Zhao, Y.; Wei, J.; Vajtai, R.; Ajayan, P.M.; Barrera, E.V. Iodine-doped carbon nanotube cables exceeding specific electrical conductivity of metals. Sci. Rep. 2011, 1, 83. [CrossRef]

41. Lee, J.-K.; Lee, S.-C.; Ahn, J.-P.; Kim, S.-C.; Wilson, J.I.B.; John, P. The Growth of AA Graphite on (111) Diamond. J. Chem. Phys. 2008, 129, 234709. [CrossRef]

42. Lee, J.-K.; Lee, S.; Kim, Y.-I.; Kim, J.-G.; Lee, K.-I.; Ahn, J.-P.; Min, B.-K.; Yu, C.-J.; Hwa Chae, K.; John, P. Structure of Multi-Wall Carbon Nanotubes: AA' Stacked Graphene Helices. Appl. Phys. Lett. 2013, 102, 161911. [CrossRef]

43. Lee, J.-K.; Kim, J.-G.; Hembram, K.P.S.S.; Kim, Y.-I.; Min, B.-K.; Park, Y.; Lee, J.-K.; Moon, D.J.; Lee, W.; Lee, S.-G.; et al. The Nature of Metastable AA' Graphite: Low Dimensional Nano- and Single-Crystalline Forms. Sci. Rep. 2016, 6, 39624. [CrossRef] [PubMed]

44. Yaya, A.; Ewels, C.P.; Suarez-Martinez, I.; Wagner, P.; Lefrant, S.; Okotrub, A.; Bulusheva, L.; Briddon, P.R. Bromination of Graphene and Graphite. Phys. Rev. B 2011, 83, 045411. [CrossRef]

45. Hof, F.; Impellizzeri, A.; Picheau, E.; Che, X.; Pénicaud, A.; Ewels, C.P. Chainlike Structure Formed in Iodine Monochloride Graphite Intercalation Compounds. J. Phys. Chem. C 2021, 125, 23383-23389. [CrossRef] 